\title{
COMMON STABILIZATIONS OF HEEGAARD SPLITTINGS OF KNOT EXTERIORS
}

\author{
Jung HoOn LEE
}

\begin{abstract}
In [4], we gave a condition for a pair of unknotting tunnels of a non-trivial tunnel number one link to give a genus three Heegaard splitting of the link complement. In this paper we prove the corresponding result for tunnel number one knots.
\end{abstract}

\section{Introduction}

For a closed 3-manifold $M$, a decomposition $M=H_{1} \cup_{S} H_{2}$, which is a gluing of two handlebodies $H_{1}$ and $H_{2}$ along their common boundary $S$, is called a Heegaard splitting of $M$.

To describe Heegaard splitting of 3-manifold with non-empty boundary, we use compression bodies instead of handlebodies. A compression body $H$ is a 3-manifold obtained from a closed surface $S$ by attaching 2-handles to $S \times I$ on $S \times\{1\}$ and capping off any resulting 2 -sphere boundary components with 3balls. $S \times\{0\}$ is denoted by $\partial_{+} H$ and $\partial H-\partial_{+} H$ is denoted by $\partial_{-} H$. Then for a 3-manifold $M$ with non-empty boundary, a Heegaard splitting $M=H_{1} \cup_{S} H_{2}$ is a gluing of two compression bodies $H_{1}$ and $H_{2}$ along their common "plus" boundary. It is known that every compact 3-manifold has a Heegaard splitting.

For a Heegaard splitting $H_{1} \cup_{S} H_{2}$, let $\alpha$ be a properly embedded arc in $H_{2}$ which is $\partial$-parallel. Attach a neighborhood of $\alpha$ in $H_{2}$ to $H_{1}$ and remove it from $H_{2}$. The result is again a Heegaard splitting $H_{1}^{\prime} \cup_{S^{\prime}} H_{2}^{\prime}$ with the genus increased by one. This operation is called a stabilization.

Suppose there are two Heegaard splittings of same genus $H_{1} \cup_{S} H_{2}$ and $H_{1}^{\prime} \cup_{S^{\prime}}$ $H_{2}^{\prime}$ for a 3-manifold $M$. The two splittings become isotopic after stabilizing both sufficiently many times [8]. However, no example has been known where actually we should stabilize more than once to make them isotopic. It has been conjectured that a single stabilization always suffices [2] (Problem 3.89) and there are some results to this direction [4], [6], [7].

Very recently, in [1] Hass, Thompson and Thurston gave examples of 3manifolds with two genus $g$ Heegaard splittings requiring $g$ stabilizations to

Received March 18, 2008; Revised November 10, 2008.

2000 Mathematics Subject Classification. 57M25, 57M50.

Key words and phrases. Heegaard splitting, stabilization, unknotting tunnel.

(C)2010 The Korean Mathematical Society 
become isotopic. We remark that the manifolds $M_{g}$ in Theorem 1.1 are closed 3-manifolds.

Theorem 1.1 ([1]). For each $g>1$ there is a 3 -manifold $M_{g}$ with two genus $g$ Heegaard splittings that require $g$ stabilizations to become equivalent.

Now we consider Heegaard splittings of knot exteriors and the stabilization problem. A tunnel system (or tunnels) of a knot (or link) $K$ in $S^{3}$ is a collection of properly embedded arcs $t_{1}, t_{2}, \ldots, t_{n}$ in the exterior of $K$ such that $H=$ $\overline{S^{3}-N\left(K \cup \bigcup_{i=1}^{n} t_{i}\right)}$ is a genus $n+1$ handlebody. The tunnel system gives rise to a Heegaard splitting of the exterior of $K$

$$
\overline{S^{3}-N(K)}=H \bigcup_{\partial H} \overline{N\left(K \cup \bigcup_{i=1}^{n} t_{i}\right)-N(K)},
$$

where $N(K)$ is in the interior of $N\left(K \cup \bigcup_{i=1}^{n} t_{i}\right)$. The minimum of such number $n$ is called the tunnel number of $K$. If the tunnel number of $K$ is 1 , the tunnel is called an unknotting tunnel for $K$.

For 3-manifolds with boundary, the stabilization conjecture can possibly fail also as in Theorem 1.1. In [3], candidates for counterexamples of genus three Heegaard splittings of knot exteriors are given.

From now we focus on the genus two case. Consider two disjoint unknotting tunnels $t_{1}$ and $t_{2}$ of a tunnel number one knot (or link) $K$. Suppose $H=$ $\overline{S^{3}-N\left(K \cup t_{1} \cup t_{2}\right)}$ is a genus three handlebody. Then by the uniqueness of Heegaard splittings of handlebody [5], $S=\partial H$ is a Heegaard surface which is a common stabilization of Heegaard splittings induced by $t_{1}$ and induced by $t_{2}$.

However $H=\overline{S^{3}-N\left(K \cup t_{1} \cup t_{2}\right)}$ may not be a handlebody, hence $\partial H$ is not a Heegaard surface even if $t_{1}$ and $t_{2}$ are isotopic tunnels. For example, take $t_{2}$ as a parallel copy of $t_{1}$. Pull a part of $t_{2}$ in a complicated way and hook it to $t_{1}$. This construction does not give a genus three Heegaard surface (Fig. 1). So there must be some restrictions on the choice of unknotting tunnels.

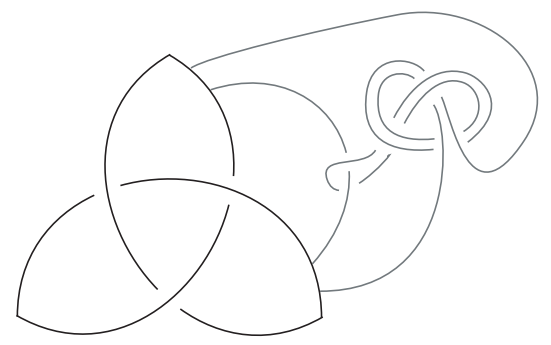

Figure 1. Embedding of tunnels which does not give a genus three splitting 
In [4], we gave a sufficient condition for a pair of unknotting tunnels of a non-trivial tunnel number one link to give a genus three Heegaard splitting of the link exterior.

Theorem $1.2([4])$. Let $L=K_{1} \cup K_{2}$ be a non-trivial tunnel number one link and $t_{1}$ and $t_{2}$ be two disjoint unknotting tunnels of $L$ such that a meridian disk $D$ of the genus two handlebody $H_{1}=\overline{S^{3}-N\left(L \cup t_{1}\right)}$ does not intersect $t_{2}$. Then $\overline{S^{3}-N\left(L \cup t_{1} \cup t_{2}\right)}$ is a genus three handlebody.

Some arguments in the proof of Theorem 1.2 worked only for 2-component links. In this paper we prove the corresponding result for tunnel number one knots.

Theorem 1.3. Let $K$ be a non-trivial tunnel number one knot and $t_{1}$ and $t_{2}$ be two disjoint unknotting tunnels of $K$ such that an essential disk $D$ of the genus two handlebody $H_{1}=\overline{S^{3}-N\left(K \cup t_{1}\right)}$ does not intersect $t_{2}$. Then $\overline{S^{3}-N\left(K \cup t_{1} \cup t_{2}\right)}$ is a genus three handlebody.

\section{Proof of Theorem 1.3}

Let $H_{2}$ be the genus two handlebody $\overline{S^{3}-N\left(K \cup t_{2}\right)} . \partial H_{1}$ (respectively $\partial H_{2}$ ) consists of two parts as in the Fig. 2 - twice punctured torus $T_{1, K}$ (respectively $T_{2, K}$ ) and an annulus $A_{1}$ (respectively $A_{2}$ ). Let $E_{1}$ and $E_{2}$ be two non-separating essential disks of $H_{2}$ which are not parallel to each other. Then $E_{1} \cup E_{2}$ cuts $H_{2}$ into a 3-ball. We may choose such $E_{1}$ and $E_{2}$ so as to satisfy $\partial T_{1, K} \cap E_{i}=\emptyset(i=1,2)$ and minimize $\left|D \cap\left(E_{1} \cup E_{2}\right)\right|$ which is the number of components of $D \cap\left(E_{1} \cup E_{2}\right)$.

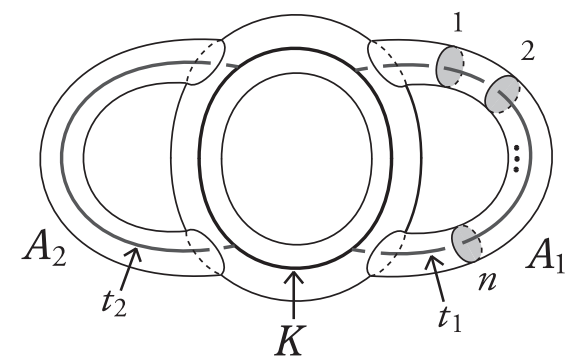

Figure 2. $N\left(K \cup t_{1}\right) \cap \operatorname{int}\left(E_{1} \cup E_{2}\right)$

Lemma 2.1. $\partial D \cap A_{1}$ and $\partial E_{i} \cap A_{2}(i=1,2)$ are non-empty. We may assume that $\partial D \cap T_{1, K}, \partial D \cap A_{1}$ consists of essential arcs and also $\partial E_{i} \cap T_{2, K}, \partial E_{i} \cap A_{2}$ $(i=1,2)$ consists of essential arcs.

Proof. Suppose $\partial D \cap A_{1}=\emptyset$. Then $\partial D$ is in $\partial N(K)$. If $\partial D$ is a meridian of $\partial N(K)$, then a punctured $S^{2} \times S^{1}$ is in $S^{3}$, a contradiction. If $\partial D$ is a 
longitude of $\partial N(K)$, then $K$ is a trivial knot, a contradiction. If $\partial D$ winds around $\partial N(K)$ longitudinally more than once, then a punctured lens space is in $S^{3}$, a contradiction. This proves $\partial D \cap A_{1} \neq \emptyset$. Similarly, $\partial E_{i} \cap A_{2} \neq \emptyset$ $(i=1,2)$.

If $\partial D$ meets $A_{1}$ in an essential loop, then a punctured $S^{2} \times S^{1}$ is in $S^{3}$, a contradiction. Therefore $\partial D \cap A_{1}$ has no essential loops. Similarly $\partial E_{i} \cap A_{2}$ $(i=1,2)$ has no essential loops.

If any of $\partial D \cap T_{1, K}, \partial D \cap A_{1}, \partial E_{i} \cap T_{2, K}, \partial E_{i} \cap A_{2}(i=1,2)$ has inessential arcs, we can remove them by isotopy. So we may assume that all the intersections are essential arcs.

Label the arcs $\partial D \cap T_{1, K}, \partial D \cap A_{1}$ of $\partial D$ with $K, t_{1}$, respectively. Also label the arcs $\partial E_{i} \cap T_{2, K}, \partial E_{i} \cap A_{2}$ with $K, t_{2}$, respectively. Let us assume that $t_{1}$ intersects $E_{1} \cup E_{2}$ transversely in $n$ points (possibly $n$ can be zero) and number the meridian disks $N\left(K \cup t_{1}\right) \cap \operatorname{int}\left(E_{1} \cup E_{2}\right)$ of $t_{1}$ consecutively along $N\left(K \cup t_{1}\right)$ (Fig. 2).

Since $D$ does not intersect $t_{2}$ by hypothesis, $D \cap\left(E_{1} \cup E_{2}\right)$ consists of loops and properly embedded arcs in $D$. If there is a loop component of $D \cap\left(E_{1} \cup E_{2}\right)$ in $D$, by cutting and pasting $E_{1} \cup E_{2}$ along the innermost disk in $D$, we can reduce $\left|D \cap\left(E_{1} \cup E_{2}\right)\right|$

So we may assume that $D \cap\left(E_{1} \cup E_{2}\right)$ consists of properly embedded arcs in $D$. An endpoint of an arc of $D \cap\left(E_{1} \cup E_{2}\right)$ which is in an arc of $\partial D$ labelled $t_{1}$ corresponds to a meridian disk $N\left(K \cup t_{1}\right) \cap \operatorname{int}\left(E_{1} \cup E_{2}\right)$ of $t_{1}$. Label that endpoint with the number given to the corresponding meridian disk. Fig. 3 shows the intersection $D \cap\left(E_{1} \cup E_{2}\right)$ and $D \cap E_{i}(i=1,2)$ on $D$ and $E_{i}$ $(i=1,2)$, respectively.
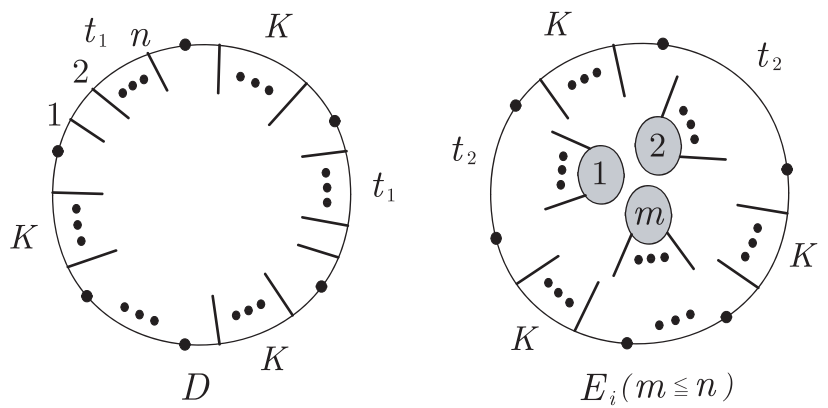

Figure 3. $D \cap\left(E_{1} \cup E_{2}\right)$ and $D \cap E_{i}(i=1,2)$ with labels

Lemma 2.2. Any arc of $\partial D$ labelled $K$ whose endpoints are in the same puncture of $T_{1, K}$ has (nonempty) intersection with $E_{1} \cup E_{2}$. 
Proof. Suppose an arc $a$ labelled $K$ whose endpoints are in the same puncture of $T_{1, K}$ has no intersection with $E_{1} \cup E_{2}$. Let $b$ be the subarc of the puncture of $T_{1, K}$ joining two endpoints of $\partial a$ (Fig. 4). Then $a \cup b$ is a closed curve.

If $a \cup b$ bounds a disk $\Delta$ in $\partial N(K)$, then $\Delta$ should contain the other puncture since $a$ is an essential arc in $T_{1, K}$. Then the number of intersection points of (one puncture of $\left.T_{1, K}\right) \cap \partial D$ and (the other puncture of $\left.T_{1, K}\right) \cap \partial D$ differs by two, a contradiction. Hence $a \cup b$ is an essential loop in $\partial N(K)$.

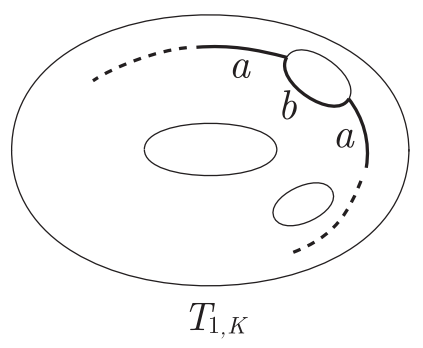

FiguRE 4. $a \cup b$ bounds a disk

Cut the genus two handlebody $H_{2}=\overline{S^{3}-N\left(K \cup t_{2}\right)}$ by $E_{1} \cup E_{2}$. Then we get a 3 -ball. Since $a \cup b$ does not intersect $E_{1} \cup E_{2}$, it bounds a properly embedded disk in the 3-ball. If $a \cup b$ is a meridian of $\partial N(K)$, a punctured $S^{2} \times S^{1}$ is embedded in $S^{3}$, a contradiction. If $a \cup b$ is a longitude of $\partial N(K)$, then $K$ is a trivial knot, a contradiction. If $a \cup b$ winds around $\partial N(K)$ longitudinally more than once, then a punctured lens space is embedded in $S^{3}$, a contradiction.

Consider an outermost arc $a$ of $D \cap\left(E_{1} \cup E_{2}\right)$ in $D$. Let $\Delta$ be the outermost disk of $D$ corresponding to $a$. Without loss of generality, we may assume that $a \subset D \cap E_{1}$. Let $\partial a=\{p, q\}$. There are several cases according to the labels of the arcs of $\partial D$ containing $p$ and $q$.

Case 1. $p$ and $q$ are in one arc labelled $t_{1}$, and the two numbers labelled to $p$ and $q$ are $i$ and $i+1$, respectively (Fig. 5).

Pushing $E_{1}$ along $\Delta$ removes $a$, so we can reduce $\left|D \cap\left(E_{1} \cup E_{2}\right)\right|$.

Case 2. $p$ and $q$ are in one arc labelled $K$ (Fig. 6).

By cutting and pasting $E_{1}$ along $\Delta$, we can get two disks $E_{1}^{\prime}$ and $E_{1}^{\prime \prime}$. By pushing slightly, we can make $E_{1}^{\prime}$ and $E_{1}^{\prime \prime}$ disjoint from $E_{1}$ and $E_{2}$.

If $E_{1}^{\prime}$ (respectively $E_{1}^{\prime \prime}$ ) is isotopic to $E_{1}$, we can reduce $\left|D \cap\left(E_{1} \cup E_{2}\right)\right|$ by replacing $E_{1}$ with $E_{1}^{\prime}$ (respectively $E_{1}^{\prime \prime}$ ). This occurs when $E_{1}^{\prime \prime}$ (respectively $\left.E_{1}^{\prime}\right)$ is $\partial$-parallel.

Suppose $E_{1}^{\prime}$ and $E_{1}^{\prime \prime}$ are not $\partial$-parallel and not isotopic to $E_{1}$. If $E_{1}^{\prime}$ (respectively $E_{1}^{\prime \prime}$ ) is isotopic to $E_{2}$, then $E_{1}^{\prime \prime}$ (respectively $E_{1}^{\prime}$ ) should be isotopic to one of the two in Fig. 7 since it cannot happen that both $E_{1}^{\prime}$ and $E_{1}^{\prime \prime}$ are 


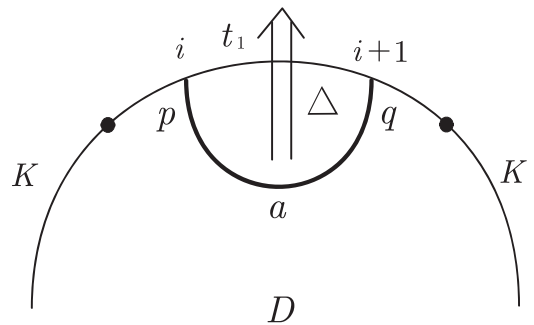

FiguRE 5. $p$ and $q$ are in one arc labelled $t_{1}$
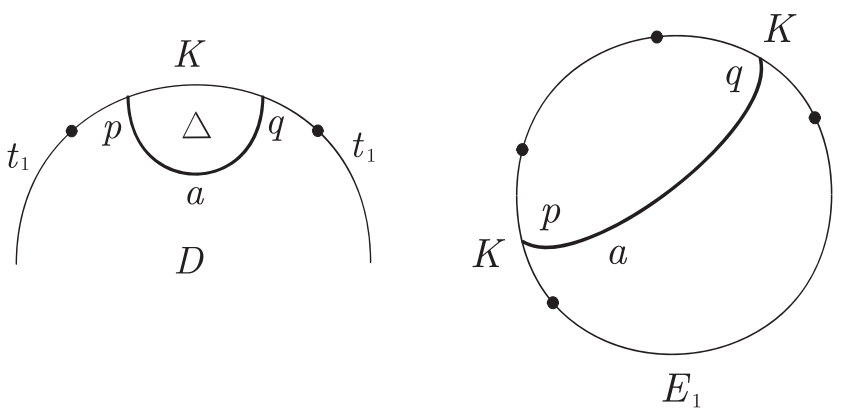

Figure $6 . p$ and $q$ are in one arc labelled $K$

isotopic to $E_{2}$. But we can observe that the left one in Fig. 7 cannot happen and in the right one in Fig. $7, E_{1}^{\prime \prime} \cup E_{2}$ (respectively $E_{1}^{\prime} \cup E_{2}$ ) cuts $H_{2}$ into a 3-ball. Therefore $\left|D \cap\left(E_{1} \cup E_{2}\right)\right|$ can be reduced by replacing $E_{1}$ with $E_{1}^{\prime \prime}$ (respectively $E_{1}^{\prime}$ ).

Now we consider the case that none of $E_{1}^{\prime}$ and $E_{1}^{\prime \prime}$ is isotopic to $E_{i}(i=1,2)$. Since $E_{1}$ is non-separating, at least one of $E_{1}^{\prime}$ and $E_{1}^{\prime \prime}$, say $E_{1}^{\prime}$, is non-separating and it is again the right one in Fig. 7. Then we can reduce $\left|D \cap\left(E_{1} \cup E_{2}\right)\right|$ by replacing $E_{1}$ with $E_{1}^{\prime}$.

In any case, we can see that the replaced $E_{1}$ and $E_{2}$ satisfy $\partial T_{1, K} \cap E_{i}=\emptyset$ $(i=1,2)$.

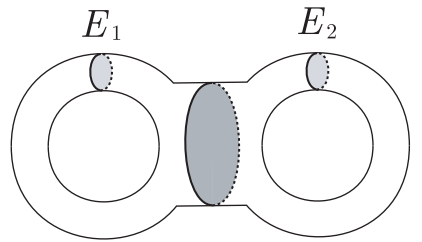

$\mathrm{H}_{2}$

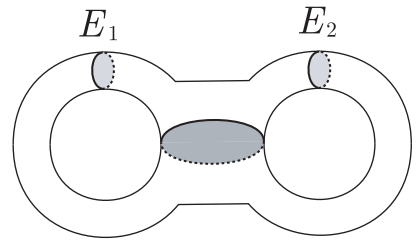

$\mathrm{H}_{2}$

Figure 7 . Two essential disks in $H_{2}$ not isotopic to $E_{i}(i=1,2)$ 
Case 3. $p$ is in an arc labelled $K$ and $q$ is in an adjacent arc labelled $t_{1}$ (Fig. 8).

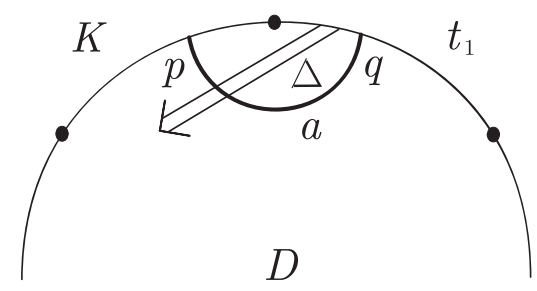

Figure 8. $p$ is in an arc labelled $K$ and $q$ is in an adjacent arc labelled $t_{1}$

Note that the number labelled to $q$ is 1 or $n$. Suppose the number labelled to $q$ is 1 . Then the meridian disk of $t_{1}$ with label 1 cuts $N\left(t_{1}\right)$ into two parts. Slide one of the parts adjacent to $\Delta$ along and then off $\Delta$. Then $\left|D \cap\left(E_{1} \cup E_{2}\right)\right|$ is reduced. It is similar in the case that the number labelled to $q$ is $n$.

Remove all possible outermost arcs of Case 1, Case 2 and Case 3. We'll show that $t_{1} \cap\left(E_{1} \cup E_{2}\right)=\emptyset$.

Lemma 2.3. Let an arc a of $D \cap\left(E_{1} \cup E_{2}\right)$ divide $D$ into two subdisks $\Delta_{1}$ and $\Delta_{2}$. Let $\partial a=\{p, q\}$. Let $p_{1}$ be one of the endpoints of the arc of $\partial D$ containing $p$ that belongs to $\Delta_{1}$ and $q_{1}$ be one of the endpoints of the arc of $\partial D$ containing $q$ that belongs to $\Delta_{1}$ (Fig. 9). Then there exists no arc a with the following properties.

- $p$ and $q$ are in different arcs of $\partial D$ labelled $t_{1}$ and both $p$ and $q$ have the same labelled number, say $m$.

- $p_{1}$ and $q_{1}$ belong to different punctures of $\partial T_{1, K}$.

Proof. Suppose such an arc $a$ exists. Without loss of generality, we may assume that $a \subset E_{1}$. The meridian disk of $N\left(t_{1}\right) \cap E_{1}$ labelled $m$ divides $N\left(t_{1}\right)$ into two parts $A$ and $B$. Let $A$ be the part containing $p_{1}$ and $B$ be the part containing $q_{1}$. If we move from $p$ to $q$ along the $\operatorname{arc} a$, we can see that the position of two parts $A$ and $B$ on both sides of the meridian disk labelled $m$ is exchanged. This is a contradiction, hence there exist no such arc $a$.

If $t_{1} \cap\left(E_{1} \cup E_{2}\right) \neq \emptyset$ after removing all possible outermost arcs of Cases 1,2 and 3 , an outermost arc $a$ with $\partial a=\{p, q\}$ would be like this $-p$ is in an arc labelled $t_{1}, q$ is in another arc labelled $t_{1}$ and there is one arc labelled $K$ between them. Furthermore, the number labelled to $p$ should be 1 and the number labelled to $q$ should be $n(n \geq 2)$, by Lemma 2.2 and Lemma 2.3.

If $n>2$, consider only the arcs of $D \cap\left(E_{1} \cup E_{2}\right)$ in $D$ which has at least one endpoint with labelled number 2 or $n-1$. Then among them, an outermost 

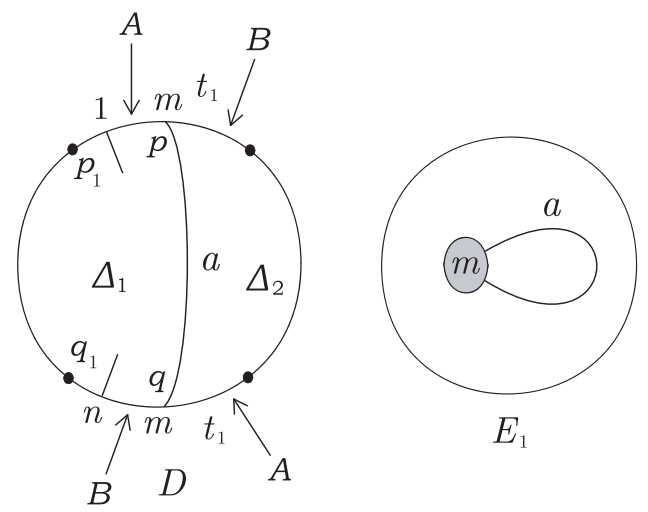

FiguRE 9. This kind of intersection does not exist

arc $a$ with $\partial a=\{p, q\}$ would be such that $p$ and $q$ with labelled number 2 and $n-1$ respectively $(n \geq 4)$ are in different arcs labelled $t_{1}$ and there is one arc labelled $K$ between them, and an arc whose endpoints have labelled number 1 and $n$ is nested, by Lemma 2.2 and Lemma 2.3. Continuing in this way, there are some nested arcs as in the Fig. 10 and $n=2 m$, an even number.

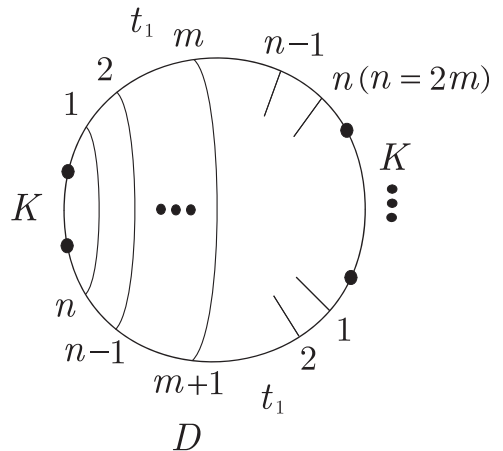

FIGURE 10. $m$ nested $\operatorname{arcs}(n=2 m)$

Now we concentrate on the $\operatorname{arcs}$ of $D \cap\left(E_{1} \cup E_{2}\right)$ in $D$ which has endpoint with labelled number $m$ or $m+1$. The claim is that such an arc has one endpoint with labelled number $m$ and the other $m+1$. The idea is that we consider only the arcs which seem to be obstacles and consider an outermost arc among them. The collection $\Gamma$ of such arcs are arcs with

- each of its endpoints is in an arc labelled $K$, or

- one endpoint has labelled number $i$, and the other has labelled number not equal to $n-i+1$ or is in an arc labelled $K(1 \leq i \leq n)$. 
Let $a$ be an outermost arc among the arcs in $\Gamma$ with $\partial a=\{p, q\}$ and $\Delta$ be the outermost disk corresponding to $a$. Note that an arc not belonging to $\Gamma$ has one endpoint with labelled number $i$ and the other $n-i+1(1 \leq i \leq n)$.

Case I. $p$ and $q$ are in the arcs labelled $K$.

Since we removed all outermost arc of Cases 1, 2 and 3, there are at least two arcs labelled $t_{1}$ in $\partial \Delta$, and at least one arc labelled $K$ having no intersection with $E_{1} \cup E_{2}$ in $\partial \Delta$. Remember that an arc labelled $K$ with no intersection with $E_{1} \cup E_{2}$ has endpoints in different punctures of $\partial T_{1, K}$ by Lemma 2.2. Cut $D$ by $E_{1} \cup E_{2}$. Then we can obtain a disk like the one in Fig. 11 in a 3-ball $B$. The boundary of the disk is an alternating sequence of arcs with endpoints labelled $m$ and $m+1$ and arcs in $\partial B$, and the appearance of $m$ and $m+1$ is alternating. But this is a contradiction because there cannot be a disk with its boundary running on the neighborhood of a curve longitudinally more than once in $S^{3}$, which results in a punctured lens space in $S^{3}$.

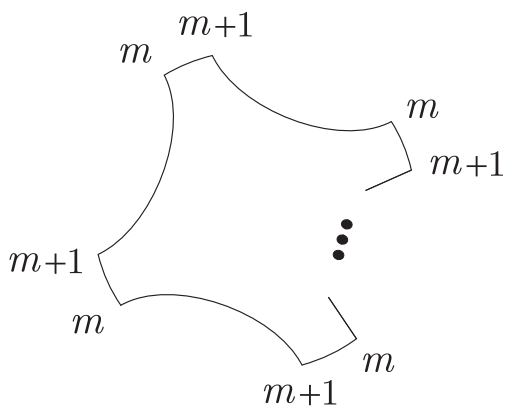

Figure 11 . One of the disks $D$ cut by $E_{1} \cup E_{2}$

Case II. $p$ is in an arc labelled $t_{1}$ and $q$ is in an arc labelled $K$.

As in the Case I, consider the disk which has labels $m$ and $m+1$ on the boundary as in the Fig. 11. If each of the labels $m$ and $m+1$ appears only once in the boundary, then the disk is the one in Fig. 5 of Case 1, which contradicts that we removed all the arcs of Cases 1, 2 and 3. If each of $m$ and $m+1$ appears more than once, it is a contradiction by the arguments of Case I.

Case III. $p$ has labelled number $i$ and $q$ has labelled number not equal to $n-i+1(1 \leq i \leq n)$.

If there is only one arc labelled $K$ in $\partial \Delta$, the numbers on the arcs labelled $t_{1}$ of $\partial \Delta$ would not be matched correctly. Otherwise, there is at least one arc of $D \cap\left(E_{1} \cup E_{2}\right)$ in $\Delta$ with one endpoint labelled $m$ and the other $m+1$. Then considering the disk like the one in Fig. 11, we get a contradiction by Case I and Case II. 
Now we may assume $t_{1} \cap\left(E_{1} \cup E_{2}\right)=\emptyset$. Let an arc $a$ with $\partial a=\{p, q\}$ be an outermost arc of $D \cap\left(E_{1} \cup E_{2}\right)$ in $D$ and $\Delta$ be the outermost disk corresponding to $a$. Obviously $p$ and $q$ are in the arcs labelled $K$. Cut $D$ by $E_{1} \cup E_{2}$. If there is at least one arc labelled $K$ having no intersection with $E_{1} \cup E_{2}$ in $\partial \Delta$, there are at least two arcs labelled $t_{1}$ in $\partial \Delta$. Then we get a contradiction on $\Delta$ by similar arguments as in the Case I.
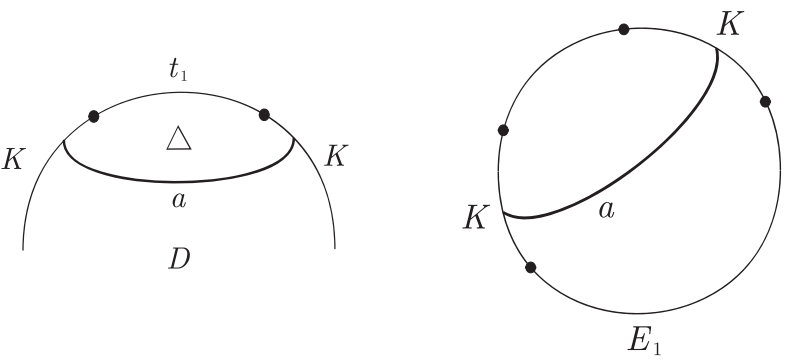

Figure 12. Stabilizing disk for $t_{1}$

If there is no arc labelled $K$ having no intersection with $E_{1} \cup E_{2}$ in $\partial \Delta$, there is one arc labelled $t_{1}$ in $\partial \Delta$. By cutting and pasting $E_{1} \cup E_{2}$ along $\Delta$, we get a stabilizing disk for $t_{1}$ (Fig. 12). This completes the proof of Theorem 1.3.

\section{References}

[1] J. Hass, A. Thompson, and W. Thurston, Stabilization of Heegaard splittings, Geom. Topol. 13 (2009), no. 4, 2029-2050.

[2] R. Kirby, Problems in low-dimensional topology, http://math.berkeley.edu/ kirby.

[3] J. H. Lee, Stabilizing Heegaard splittings of composite tunnel number two knots, J. Knot Theory Ramifications 17 (2008), no. 8, 1035-1041.

[4] J. H. Lee and G. T. Jin, Common stabilizations of Heegaard splittings of link exteriors, Topology Appl. 150 (2005), no. 1-3, 101-110.

[5] M. Scharlemann and A. Thompson, Heegaard splittings of (surface) $\times I$ are standard, Math. Ann. 295 (1993), no. 3, 549-564.

[6] J. Schultens, The stabilization problem for Heegaard splittings of Seifert fibered spaces, Topology Appl. 73 (1996), no. 2, 133-139.

[7] E. Sedgwick, An infinite collection of Heegaard splittings that are equivalent after one stabilization, Math. Ann. 308 (1997), no. 1, 65-72.

[8] J. Singer, Three-dimensional manifolds and their Heegaard diagrams, Trans. Amer. Math. Soc. 35 (1933), no. 1, 88-111.

School of Mathematics

Korea Institute for Advanced Study

SEOUl 130-722, KoreA

E-mail address: jhlee@kias.re.kr 